\title{
EVALUACION TEÓRICA DE NUEVOS DERIVADOS NITRATOXICARBONO DE TETRAEDRANO
}

\author{
Luis G. Calvo1, Rodolfo Pumachagua1*
}

\begin{abstract}
RESUMEN
Se ha realizado el estudio teórico de derivados nitratoxicarbono de tetraedrano empleando los métodos: Hartree-Fock y la Teoría del funcional de la densidad. Determinamos la estructura, las propiedades electrónicas y el perfil energético; así también el análisis poblacional de Mulliken para mostrar que el incremento de grupos nitratoxicarbono disminuye las cargas de los oxígenos del grupo sustituyente.

Palabras clave: Nitratoxicarbono, tetraedrano, hidrocarburo platónico, B3LYP.

\section{THEORETICAL EVALUATION OF NEW DERIVATIVES OF THE TETRAHEDRANE NITRATOXYCARBON}

\begin{abstract}
We calculated the properties of nitratoxycarbon tetrahedrane derivatives using the methods: Hartree-Fock and density functional theory. We assessed structure, the electronic properties and energy profile to show Mulliken population analysis that the increase of nitratoxycarbon groups reduce the burdens of the oxygen's of the group substituent.
\end{abstract}

Key words: Nitratoxycarbon, tetrahedrane, platonic hydrocarbon, B3LYP.

\section{INTRODUCCIÓN}

El tetraedrano ${ }^{1,2}$ (figura 1) es un hidrocarburo platónico con cuatro átomos de carbono formando los vértices de un sistema tetraédrico. El esqueleto de carbono encierra una cavidad en forma de jaula, el ángulo de 60 grados permite almacenar una gran cantidad de energía en estos enlaces. Es interesante por al menos cuatro razones: es un hidrocarburo platónico, es una prueba del límite estructural en la química orgánica, constituye un desafío para la síntesis y es un ejemplo de química exótica ${ }^{3,4}$.

Los materiales con alta capacidad energética son los compuestos en forma de jaula con alta tensión angular y torsional; al sustituir sus hidrógenos por grupos nitrados obtienen propiedades explosivas5 muy potentes, como el tetranitrocubano ${ }^{6-9}$ o el trinitrotolueno. Por tanto, es importante conocer las propiedades electrónicas y estructurales previas a la síntesis ${ }^{10}$.

\footnotetext{
${ }^{1}$ Universidad Nacional Federico Villarreal, Facultad de Ciencias Naturales y Matemáticas, Laboratorio de Química Teórica, Jr. Chepen s/n (El Agustino) Perú. rpumachagua@gmail.com
} 


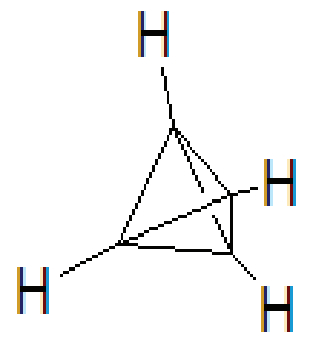

Figura 1. Tetraedrano

Diversos derivados nitro sobre el tetraedrano han sido estudiados11. El grupo nitratoxicarbono 12 mostrado en la figura 2 ha sido planteado recientemente13. Por otro lado, el grupo nitratoxicarbono y los grupos nitro presentan características similares: el intercambio electrónico en los átomos de nitrógeno y oxígeno puede ser un factor estabilizante en los sistemas donde ellos participan; así también, cuando se incrementa el número de los sustituyentes (figura 3).

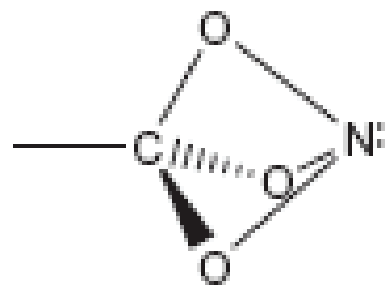

Figura 2. Grupo nitratoxicarbono

El interés en estos sistemas es la presencia del grupo nitratoxicarbono dentro de la molécula tetraedrano, las dimensiones del enlace, así como el perfil energético de la molécula y las frecuencias vibratorias permitirán plantear su estabilidad. Reportamos los resultados empleando los métodos Hartree-Fock (HF) y la de la Teoría del funcional de la densidad (DFT) empleando la función base 6-31G.

\section{ASPECTO COMPUTACIONAL}

Todos los cálculos se llevaron a cabo con el programa GAUSSIAN 09 usando la función base 6-31G con los métodos: Hartree-Fock y DFT utilizando el funcional híbrido B3LYP. Las frecuencias vibratorias se calcularon para cada molécula con ambos métodos para obtener estructuras estables. 


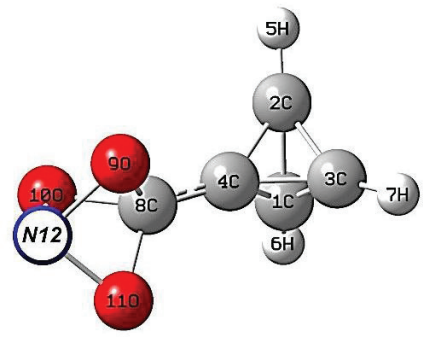

S1

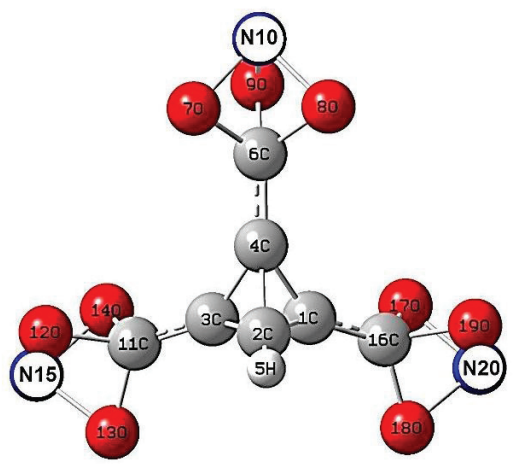

S3
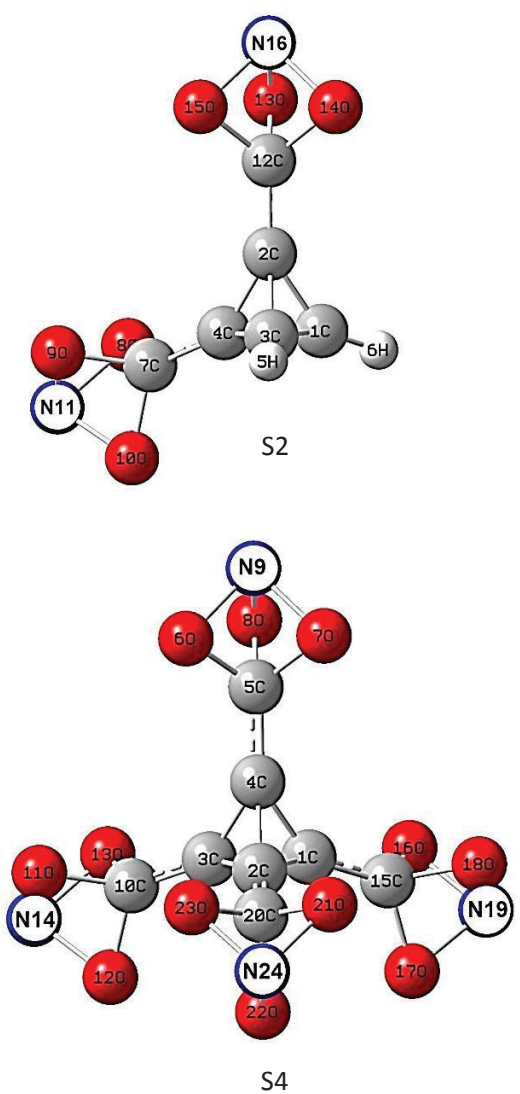

Figura 3. Estructuras de los derivados nitratoxicarbono de tetraedrano: S1, S2, S3 y S4.

\section{Perfil energético}

Tabla 1. Energía total por los métodos HF y B3LYP de los sistemas: S1, S2, S3 y S4.

\begin{tabular}{lcc}
\hline \multicolumn{1}{c}{ Moléculas en estudio } & \multicolumn{2}{c}{ Energía } \\
& HF/6-31G & B3LYP/6-31G \\
Nitratoxicarbono tetraedrano (S1) & $-469,352$ & $-472,120$ \\
Dinitratoxicarbono tetraedrano (S2) & $-785,182$ & $-789,652$ \\
Trinitratoxicarbono tetraedrano (S3) & $-1101,000$ & $-1107,176$ \\
Tetranitratoxicarbono tetraedrano (S4) & $-1416,811$ & $-1424,694$ \\
\hline
\end{tabular}




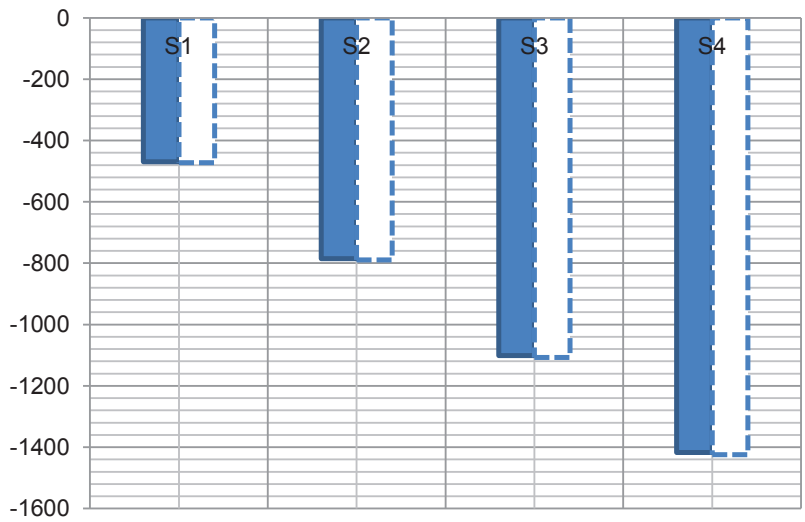

DHF/6-31G

른 $3 L Y P / 6-31 G$

Figura 4. Perfil energético por los métodos HF y B3LYP de los sistemas: S1, S2, S3 y S4.

\section{Análisis poblacional de Mulliken}

Tabla 2. Cargas promedio de oxígeno, carbono y nitrógeno en el grupo nitratoxicarbono por los métodos HF y B3LYP.

\begin{tabular}{lccc|ccc}
\hline & & & \multicolumn{3}{c}{ B3LYP/6-31 G } \\
& Oxígeno & Carbono & Nitrógeno & Oxígeno & Carbono & Nitrógeno \\
(S1) & $-0,364$ & 0,584 & 0,342 & $-0,253$ & 0,260 & 0,216 \\
(S2) & $-0,355$ & 0,574 & 0,327 & $-0,241$ & 0,251 & 0,238 \\
(S3) & $-0,343$ & 0,561 & 0,310 & $-0,231$ & 0,240 & 0,255 \\
(S4) & $-0,335$ & 0,547 & 0,288 & $-0,224$ & 0,228 & 0,269 \\
\hline
\end{tabular}

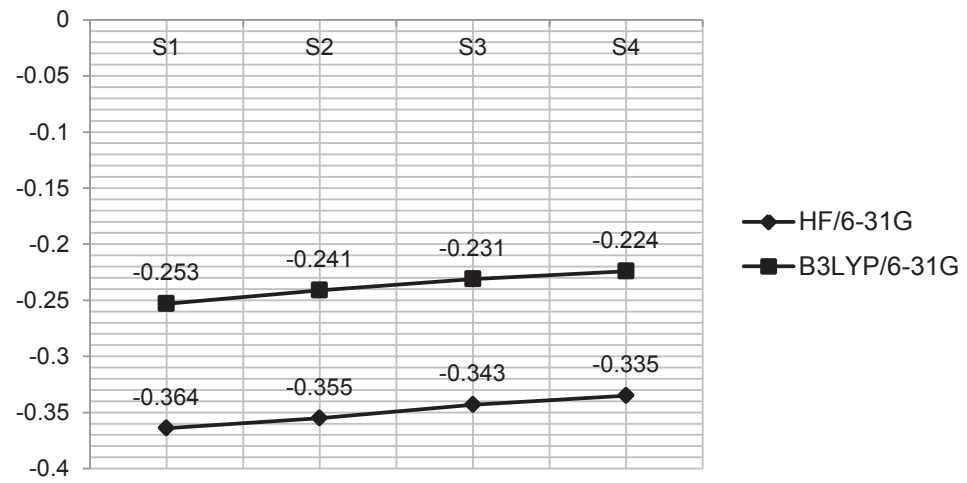

Figura 5. Carga promedio de oxígeno por los métodos HF y B3LYP versus cantidad de grupo sustituyente nitratoxicarbono. 


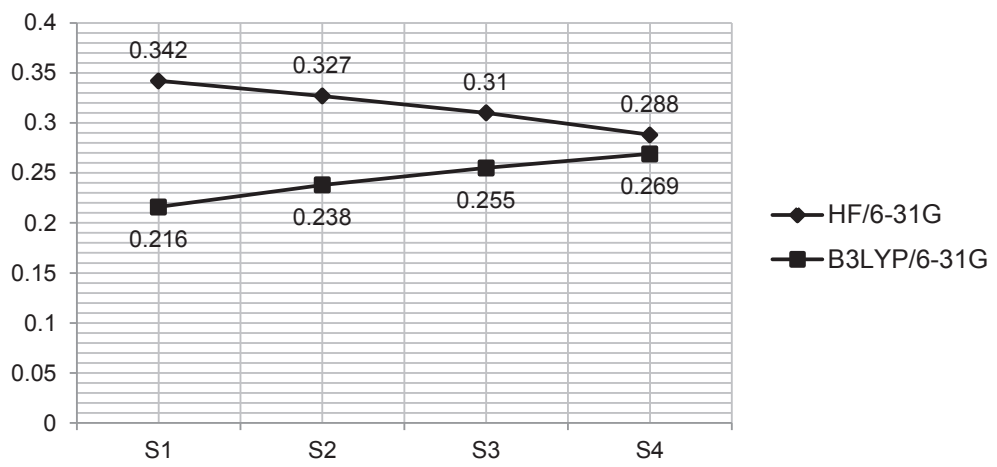

Figura 6. Carga promedio de nitrógeno por los métodos HF y B3LYP versus cantidad de grupo sustituyente nitratoxicarbono.

\section{Propiedades estructurales}

Tabla 3. Longitud de enlace C-C por los métodos HF y B3LYP de los sistemas: S1, S2, S3 y S4.

\begin{tabular}{ccccc}
\hline & \multicolumn{4}{c}{$\mathrm{HF} / 6-31 \mathrm{G}$} \\
& $\mathbf{S 1}$ & $\mathbf{S 2}$ & $\mathbf{S 3}$ & $\mathbf{S 4}$ \\
$\mathrm{C} 1-\mathrm{C} 2$ & 1,47721 & 1,48460 & 1,47771 & 1,47915 \\
$\mathrm{C} 1-\mathrm{C} 3$ & 1,47715 & 1,46766 & 1,48205 & 1,47914 \\
$\mathrm{C} 1-\mathrm{C} 4$ & 1,48838 & 1,48461 & 1,48221 & 1,47914 \\
$\mathrm{C} 2-\mathrm{C} 3$ & 1,47720 & 1,48461 & 1,47772 & 1,47914 \\
$\mathrm{C} 2-\mathrm{C} 4$ & 1,48832 & 1,48107 & 1,47754 & 1,47912 \\
$\mathrm{C} 3-\mathrm{C} 4$ & 1,48838 & 1,48460 & 1,48220 & 1,47913 \\
\hline
\end{tabular}

Tabla 4. Longitud de enlace C-C por los métodos HF y B3LYP de los sistemas: S1, S2, S3 y S4.

\begin{tabular}{ccccc}
\hline & \multicolumn{4}{c}{ B3LYP/6-31G } \\
& S1 & S2 & S3 & S4 \\
C1-C2 & 1,49250 & 1,50156 & 1,49524 & 1,49926 \\
C1-C3 & 1,49247 & 1,48349 & 1,50228 & 1,49931 \\
C1-C4 & 1,50480 & 1,50156 & 1,50229 & 1,49928 \\
C2-C3 & 1,49252 & 1,50156 & 1,49514 & 1,49937 \\
C2-C4 & 1,50474 & 1,50086 & 1,49519 & 1,49929 \\
C3-C4 & 1,50479 & 1,50156 & 1,50239 & 1,49937 \\
\hline
\end{tabular}

Tabla 5. Longitud de enlace promedio C-C del sustituyente nitratoxicarbono y tetraedrano por los métodos HF y B3LYP

\begin{tabular}{ccc}
\hline & HF/6-31G & B3LYP/6-31G \\
S1 & 1,40918 & 1,40971 \\
S2 & 1,41812 & 1,42028 \\
S3 & 1,42508 & 1,42822 \\
S4 & 1,43063 & 1,43362 \\
\hline
\end{tabular}




\section{Propiedades electrónicas}

Tabla 6. Propiedades electrónicas por los métodos HF y B3LYP de los sistemas: S1, S2, S3 y S4.

\begin{tabular}{|c|c|c|c|c|}
\hline \multicolumn{5}{|c|}{$\mathrm{HF} / 6-31 \mathrm{G}$} \\
\hline & S1 & $\mathbf{S 2}$ & S3 & S4 \\
\hline E (HOMO) & $-0,39559$ & $-0,43878$ & $-0,47998$ & $-0,51156$ \\
\hline E (LUMO) & 0,16391 & 0,1128 & 0,07997 & 0,06094 \\
\hline Energía gap & 0,5595 & 0,55158 & 0,55995 & 0,5725 \\
\hline Potencial ionización (I) & 0,39559 & 0,43878 & 0,47998 & 0,51156 \\
\hline Afinidad electrónica (A) & $-0,16391$ & $-0,1128$ & $-0,07997$ & $-0,06094$ \\
\hline Dureza global (文) & 0,27975 & 0,27579 & 0,279975 & 0,28625 \\
\hline Potencial químico $(\boldsymbol{\mu})$ & $-0,11584$ & $-0,16299$ & $-0,20001$ & $-0,22531$ \\
\hline Electrofilia global $(\boldsymbol{\omega})$ & 0,024 & 0,0482 & 0,07144 & 0,0887 \\
\hline \multicolumn{5}{|c|}{ B3LYP/6-31G } \\
\hline & S1 & S2 & S3 & S4 \\
\hline E (HOMO) & $-0,28119$ & $-0,3099$ & $-0,3263$ & $-0,33893$ \\
\hline E (LUMO) & $-0,05095$ & $-0,08134$ & $-0,10113$ & $-0,11537$ \\
\hline Energía gap & 0,23024 & 0,22856 & 0,22517 & 0,22356 \\
\hline Potencial ionización (I) & 0,28119 & 0,3099 & 0,3263 & 0,33893 \\
\hline Afinidad electrónica (A) & 0,05095 & 0,08134 & 0,10113 & 0,11537 \\
\hline 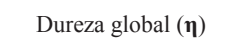 & 0,11512 & 0,11428 & 0,112585 & 0,11178 \\
\hline Potencial químico $(\boldsymbol{\mu})$ & $-0,16607$ & $-0,19562$ & $-0,213715$ & $-0,2272$ \\
\hline Electrofilia global $(\boldsymbol{\omega})$ & 0,1198 & 0,1674 & 0,2028 & 0,2308 \\
\hline
\end{tabular}
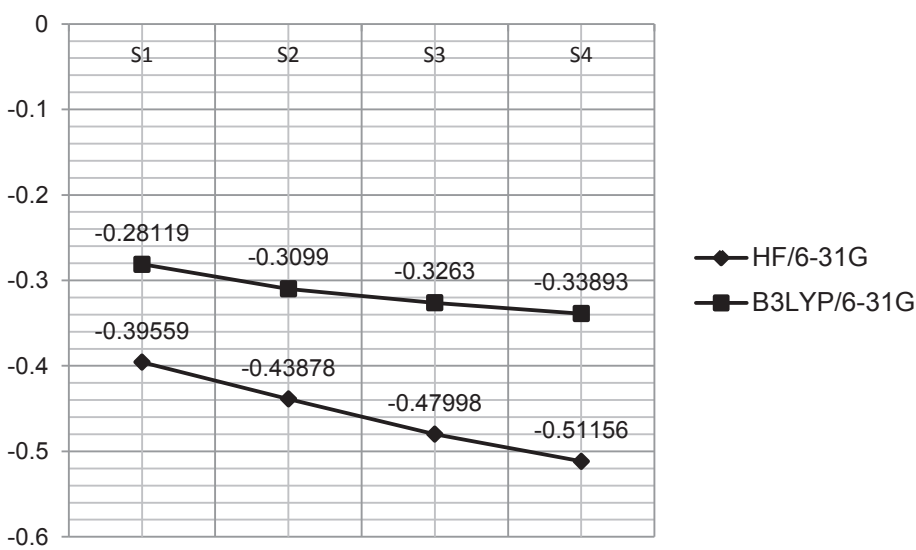

Figura 7. Energía del orbital molecular ocupado más alto (HOMO) por los métodos HF y B3LYP 


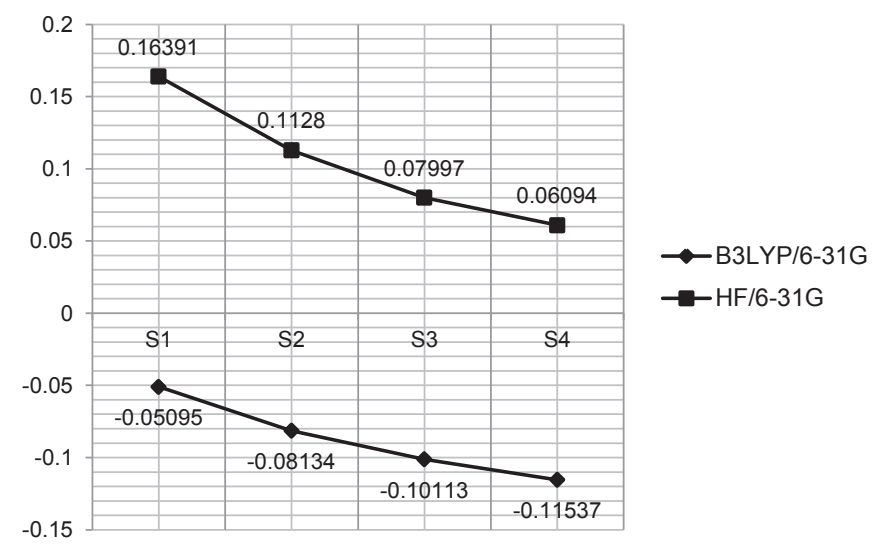

Figura 8. Energía del orbital molecular desocupado más bajo (LUMO) por los métodos HF y B3LYP

\section{Perfil energético}

\section{RESULTADOS Y DISCUSIÓN}

La geometría de equilibrio de las moléculas viene definido por una serie de coordenadas de los núcleos que permiten alcanzar un mínimo energético. Los sistemas derivados nitratoxicarbono del tetraedrano S1, S2, S3 y S4 en la tabla 1 muestran un descenso considerable de energía; la energía del $\mathbf{S 4}$ es la más baja de todos; observamos que mientras mayor es la cantidad de sustituyentes, la energía disminuye en ambos métodos de cálculo. Los valores más bajos son determinados por la teoría del funcional de la densidad.

\section{Análisis poblacional de Mulliken}

El análisis poblacional de Mulliken es un esquema arbitrario para asignar cargas de los átomos que forman una molécula. De la tabla 2 observamos una ligera disminución de carga negativa en los átomos de oxígeno conforme aumenta el número de sustituyentes nitratoxicarbono en el tetraedrano. El método Hartree-Fock presenta los valores de carga más bajos para el átomo de oxígeno. La pérdida de carga negativa en el oxígeno reduce las repulsiones electrostáticas entre ellos, dentro del sistema tetraédrico estableciendo las conformaciones más estables de los distintos sistemas.

El átomo de nitrógeno muestra un comportamiento distinto. El método Hartree-Fock presenta una disminución de carga, ocasionando un ligero desequilibrio en las atracciones electrostáticas, porque tanto el oxígeno como el nitrógeno están perdiendo carga. Sin embargo, en DFT hay un incremento en la carga del nitrógeno que no afectaría en gran medida a las atracciones electrostáticas nitrógeno-oxígeno por una redistribución de la carga. El átomo de carbono une el sistema tetraédrico y el sustituyente muestra un comportamiento igual que los átomos de oxígeno en los dos métodos de cálculo.

\section{Propiedades estructurales}

La geometría molecular es un indicador de las interacciones intra e inter-moleculares de 
cualquiera estructura química. En las tablas 3 y 4 observamos que al aumentar los grupos sustituyentes nitratoxicarbono hay un equilibrio significativo en las longitudes de enlaces del tetraedrano; dicho comportamiento se puede observar con cada sustitución.

En la tabla 5 se observa que el enlace carbono-carbono entre sustituyente y el sistema tetraedrano se va alargando conforme aumenta el número de sustituciones. Este comportamiento se confirma por ambos métodos de cálculo, desde la longitud de 1,40971 ̊ a 1,43362 Å con el

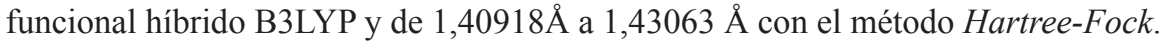

Las longitudes de enlace carbono-carbono obtenidas en los derivados nitratoxicarbono del tetraedrano mantienen valores muy cercanos, a diferencia de los valores obtenidos sobre la geometría molecular del tetranitrotetraedrano reportado en la literatura ${ }^{11}$. La introducción de los grupos nitro debilita los enlaces en el tetraedrano, incrementando la longitud en algunos enlaces carbono-carbono, llegando a medir $1,773 \AA$ y $1,770 \AA$, ocasionando la ruptura de los enlaces.

\section{Propiedades electrónicas}

Las propiedades electrónicas en sistemas orgánicos e inorgánicos dependen de los orbitales de frontera HOMO y LUMO. Los orbitales moleculares se extienden entre todos los átomos de una molécula y son combinaciones lineales de orbitales atómicos, los orbitales moleculares que interactúan son generalmente el de más alta energía ocupado HOMO y el de más baja energía desocupado LUMO. La tabla 6 muestra que la brecha de energía HOMOLUMO denominado energía gap muestra una ligera disminución en relación al incremento de las sustituciones del grupo nitratoxicarbono desde 0,23024 en el sistema $\mathbf{S 1}$ a 0,22356 en S4 dentro del contexto del método DFT; en el método de Hartree-Fock sucede de forma contraria; el sistema $\mathbf{S} 1$ muestra 0,5595 y el $\mathbf{S} 4$ 0,5725. La energía gap refleja la estabilidad cinética, la reactividad química, la polarizabilidad molecular y la dureza o blandura14. Los métodos de correlación electrónica mejoran los valores sobre los obtenidos por el método de Hartree-Fock.

El potencial de ionización (I) expresado como $\mathbf{I}=-$ EHOMO es superior dentro del método Hartree-Fock con 0,51156 y empleando el funcional B3LYP obtenemos un valor de 0,33893, con una diferencia de 0,17263 en ambos métodos.

La afinidad electrónica (A) expresada como A = -ELUMO muestra valores altos, característico en sistemas que pueden acomodar fácilmente un exceso de electrones provenientes de los alrededores. Los mejores valores de energía del orbital molecular desocupado más bajo LUMO son obtenidos en la teoría DFT.

La dureza química ( $\boldsymbol{\eta}$ ) expresado como $\boldsymbol{\eta}=1 / 2($ ELUMO - EHOMO) es una propiedad global del sistema y mide la resistencia impuesta por éste al cambio en su distribución electrónica. En este contexto la dureza es un descriptor de la reactividad mostrando valores mayores en el método Hartree-Fock.

El potencial químico $(\boldsymbol{\mu})$ expresado como $\boldsymbol{\mu}=1 / 2($ EHOMO - ELUMO) es una propiedad donde los electrones tienden a fluir de regiones de baja hacia alta electronegatividad (de regiones de alto potencial químico a regiones de bajo potencial químico). Observamos en la tabla 6 una tendencia que se va acentuando hasta llegar a sustituir todos los hidrógenos por grupos nitratoxicarbono, obteniendo así -0,225 y -0,227 en el método Hartree-Fock y B3LYP 
respectivamente, siendo el sistema $\mathbf{S} 4$ el que presenta el menor potencial químico.

En cuanto a la electrofilia global $(\boldsymbol{\omega})$ expresado como $\boldsymbol{\omega}=\mu 2 / 2 \eta$, el sistema $\mathbf{S} 4$ entrega un valor de 0,2308 en la teoría del funcional de la densidad siendo el más representativo en esta serie.

\section{CONCLUSIONES}

El tetraedrano y sus derivados nitratoxicarbono son teóricamente estudiados a un nivel de cálculo HF/6-31G y B3LYP/6-31G.

El incremento de grupos sustituyentes nitratoxicarbono en el tetraedrano contribuye a la estabilidad de la molécula.

El grupo nitratoxicarbono presenta menor redistribución de carga en los átomos de oxígeno, respecto a los grupos nitro.

\section{AGRADECIMIENTO}

Los autores agradecen al Dr. William Tiznado Vásquez de la Universidad Andrés Bello, Santiago de Chile, por brindarnos soporte computacional.

\section{BIBLIOGRAFÍA}

1. A. Greenberg \& J. F. Liebman, Organic Chemistry, A Series of Monographs, Vol. 38: Strained Organic Molecules, H. H. Wasserman (editor), Academic Press, Inc., U.S.A., 1978, 85-90.

2. G. Maier, Tetrahedrane and Cyclobutadiene. Angew. Chem. Int. Ed. Engl., 1988; 27: 309-332.

3. E. G. Lewars, Modeling Marvels, Computational Anticipation of Novel Molecules, Springer Science+Business Media B.V., 2008, 81-104.

4. B. A. Hess, Jr. \& L. J. Schaad, Ab Initio Second-Order Møller-Plesset Calculation of the Vibrational Spectrum of Tetrahedrane. J. Am. Chem. Soc., 1985; 107: 865-866.

5. J. Akhavan, the Chemistry of Explosives (RSC Paperbacks), The Royal Society of Chemistry, 1998, 18-43.

6. G. W. Griffin \& A. P. Marchand, Synthesis and Chemistry of Cubanes. Chem. Rev., 1989; 89: 997-1010.

7. P. E. Eaton, R. L. Gilardi \& M.-X. Zhang, Polynitrocubanes: Advanced High-Density, High-Energy Materials. Adv. Mater, 2000; 12: 1143-1148.

8. R. M. Richard \& D. W. Ball, B3LYP Calculations on the Thermodynamic Properties of a Series of Nitroxycubanes Having the Formula $\mathrm{C}_{8} \mathrm{H}_{8}-\mathrm{x}\left(\mathrm{NO}_{3}\right)_{\mathrm{x}}(\mathrm{x}=1-8)$, Journal of Hazardous Materials. 2009; 164: 1595-1600.

9. D. A. Hrovat, W. T. Borden, P. E. Eaton \& B. Kahr, A Computational Study of the Interactions Among the Nitro Groups in Octanitrocubane. J. Am. Chem. Soc., 2001; 123: 1289-1293.

10. A. Nemirowski, H. P. Reisenauer \& P. R. Schreiner, Tetrahedrane-Dossier of an Unknown. Chem. Eur. J., 2006; 12: 7411-7420.

11. G. Zhou, J.-L. Zhang, N.-B. Wong \& A. Tian, Computational Studies on a Kind of Novel Energetic Materials Tetrahedrane and Nitro Derivatives. Journal of Molecular Structure (Theochem), 2004; 668: 189-195. 
12. R. W. Zoellner, C. L. Lazen \& K. M. Boehr, A Computational Study of Novel Nitratoxycarbon, Nitritocarbonyl, and Nitrate Compounds and their Potential as High Energy Materials. Computational and Theoretical Chemistry, 2012; 979: 33-37.

13. R. J. Buszek, C. M. Lindsay \& J. A. Boatz, Tetrakis (nitratoxycarbon) Methane (Née CLL-1) as a Potential Explosive Ingredient: a Theoretical Study. Propellants Explos. Pyrotech., 2010; 35: 1-5.

14. D. Pegu \& N. B. Singh, Quantum Chemical Calculations of Molecular Structure, Electronic, Thermodynamic and Non-linear optical properties of 2-amino-3nitro-6methyl pyridine. International Journal of Advanced Research, 2013; 1: 531-538. 